\title{
Asbesto, asbestose e câncer: critérios diagnósticos"
}

\author{
VERA LUIZA CAPELOZZI ${ }^{1}$
}

As doenças asbesto-induzidas constituem um grave problema de saúde em decorrência de grande número de trabalhadores expostos ao asbesto ao longo dos últimos 50 anos. Processos judiciais contra indústrias que lidam com asbesto somam centenas, com crescente adição de novos casos. $\mathrm{O}$ assunto relativo à asbestose é complexo e, muito embora a história natural das doenças induzidas esteja bem estabelecida, muitas áreas importantes, como a patologia, permanecem ainda pouco compreendidas. No Brasil, desde 1940, o asbesto é explorado comercialmente e nos últimos anos sua produção foi da ordem de 200.000 toneladas por ano, estimando-se que na atividade de mineração cerca de 10.000

trabalhadores foram expostos a essa fibra, desconhecendo-se a estimativa do número de pessoas expostas na produção de fibrocimento, especialmente telhas e caixas d'água. Um estudo, com metodologia de investigação científica apropriada, para avaliar as repercussões sobre a saúde dos

trabalhadores nas minas de asbesto em nosso país, foi elaborado e intitulado "Morbidade e mortalidade entre trabalhadores expostos ao asbesto na atividade de mineração 1940-1996", de cunho interinstitucional. O objetivo deste trabalho foi fornecer uma visão ampla das doenças asbestoinduzidas, com ênfase nas dificuldades no diagnóstico histopatológico, através da experiência adquirida com o desenrolar desse projeto. (J Pneumol 2001;27(4):206-218)

\section{Asbestos, asbestosis and cancer: diagnostic criteria}

Asbestos-induced diseases are still major health problems, as a remarkably large number of workers have been exposed to asbestos over the past 50 years. Personal injury lawsuits against asbestos manufacturers number hundreds of thousands, and new cases are still being filed. Asbestosis is a complex issue, and although the broad outlines of asbestos-related diseases are well set, many important areas, especially pathology, are poorly understood. In Brazil, since 1940, asbestos has been commercially explored, producing around 200,000 tons/year, exposing about 10,000 workers in the mining activity, and an unknown number of workers in asbestoscement industry. A study, with scientific appropriate investigation, with the purpose to evaluate the effects of asbestos exposure over the health of the mine workers in this country, was done by interinstitution researchers, and untitled "Morbidity and mortality among workers exposed to asbestos in mining activities - 1940/1996". With the experience achieved in the course of this study, the objective of this report is to make an overview of asbestos-related diseases, mainly with respect to difficulties in establishing the histopathologic diagnosis.

\footnotetext{
* Parte das informações contidas nesta revisão foram adquiridas no desenrolar do Projeto "Morbidade e mortalidade entre trabalhadores expostos ao asbesto na atividade de mineração - 1940-1996" Projeto temático Fapesp - Processo n 96/10415-6. Projeto interinstitucional, com a participação de: Vera Luiza Capelozzi (Departamento de Patologia - FMUSP), Jorge Kawakama (Divisão de Doenças Respiratórias - Incor - FMUSP), Mário Terra Filho (Divisão de Doenças Respiratórias - Incor - FMUSP), Reinaldo Tavares Rodrigues (Disciplina de Radiologia - Unifesp), Luiz Eduardo Nery (Disciplina de Radiologia - Unifesp), Ericsson Bagatin (Área de Saúde Ocupacional e Disciplina de Pneumologia - Unicamp). Apoio Científico: Fapesp.
}

1. Departamento de Patologia - FMUSP.

Recebido para publicação em 11/9/00. Aprovado, após revisão, em 13/3/01.
Descritores - Patologia. Asbestose. Neoplasias. Diagnóstico.

Key words - Asbestosis. Pathology. Neoplasms. Diagnosis.

\section{INTRODUÇÃO}

\section{Considerações gerais}

As doenças pulmonares ocupacionais apresentam elevados índices de prevalência em todo o mundo. No Brasil, desconhecemos não somente índices epidemiológicos, como também de prevalência, incidência ou ocorrência das pneumoconioses. Nesse contexto, dispensamse comentários em relação ao custo econômico e social.

Ainda que a silicose e a asma ocupacional sejam as mais conhecidas doenças respiratórias em nosso meio, 
as doenças relacionadas ao asbesto (DRA) vêm sendo diagnosticadas em crescente número no Brasil, levando com freqüência o pneumologista a opinar sobre o tema, seja na esfera clínica, ocupacional e legal.

$\mathrm{O}$ asbesto ou amianto é uma fibra mineral, encontrada naturalmente na crosta terrestre, do grupo dos silicatos hidratados, flexível, praticamente indestrutivel, altamente resistente ao calor e conhecido desde a antigüidade. Os gregos usavam como mecha das tochas dos templos e na produção de cerâmicas ${ }^{(1,2)}$.

Sua exploração comercial começa em 1878 na região de Quebec, no Canadá, e desde então sua produção mundial atingiu cerca de 4 milhões de toneladas nos últimos anos. Devido a suas propriedades de incombustibilidade, flexibilidade, boa resistência à tensão e corrosão, excelente isolante térmico e acústico, foi utilizado em aproximadamente 3.000 aplicações, desde produção de caixas d'água e telhas de fibrocimento, na fabricação de lonas, pastilhas e componentes de fricção, como isolante térmico em caldeiras, fornos, tubulações, na confecção de roupas, mangueiras à prova de fogo, entre tantos outros. Do exposto, depreende-se o elevado número de trabalhadores expostos nesses ambientes de trabalho, bem como aqueles da atividade de mineração e beneficiamento ${ }^{(1-3)}$.

Geologicamente, o asbesto é derivado de dois tipos de rocha-mãe: o serpentinito e o anfibólio, ambos silicatos fibrosos com diferenças físico-químicas específicas. Deriva-se do serpentinito, a fibra crisotila (asbesto branco), a mais utilizada no mundo. O Brasil faz extração de crisotila e sua produção atinge aproximadamente 200.000 toneladas/ano. Entre os anfibólios existe a crocidolita (asbesto azul), a amosita (asbesto marrom), a antofilita e a tremolita. Essas fibras vêm sofrendo restrições quanto ao seu uso devido a sua estreita relação de causalidade com as DRA, principalmente o câncer de pulmão $e$ asbestose $e^{(4)}$.

A exposição ocupacional $e$ ambiental a essas fibras pode acarretar uma série de doenças, especialmente para o aparelho respiratório: a asbestose, alterações pleurais benignas, câncer de pulmão e o mesotelioma de pleura e peritônio(5).

A exposição ocupacional ao asbesto é um fato amplamente constatado em todos os países industrializados $e$ que ainda persiste nos dias atuais. Em entrevistas detalhadas, cerca de 20 a $40 \%$ de adultos homens relatam algum tipo de trabalho no passado em que tiveram contato com asbesto. No Leste Europeu, na América do Norte, Japão e Austrália o uso do asbesto teve pico nos anos 70. Conseqüentemente, é estimado ocorrerem cerca de 10.000 mesoteliomas e 20.000 casos de câncer de pulmão asbesto-induzido por ano em uma população de aproximadamente 800 milhões de pessoas ${ }^{(6)}$.

No Brasil, desde 1940, o asbesto é explorado comercialmente. Estima-se que na atividade de mineração cer-
Siglas e abreviaturas utilizadas neste trabalho

DRA - Doenças relacionadas ao asbesto

TC - Tomografia computadorizada

TCAR - Tomografia computadorizada de alta resolução

CA - Corpos de asbesto

ME - Microscopia eletrônica

UIP - Pneumonia intersticial usual

BOOP - Bronquiolite obliterante com pneumonia organizante DIP - Pneumonia intersticial descamativa

ca de 10.000 trabalhadores foram expostos a essa fibra. Em torno de $90 \%$ da produção é utilizada na fabricação de fibrocimento, especialmente telhas e caixas d'água, desconhecendo-se a estimativa do número de pessoas expostas nesse ramo de atividade. Algumas informações de jornais e revistas não especializadas citam entre 200.000 e 300.000 indivíduos.

Mesmo utilizando essa fibra desde a década de 40 e com grande número de trabalhadores com exposição a esse mineral, não existe nenhum estudo epidemiológico, com metodologia de investigação científica apropriada, para estimar os perfis sobre a saúde dos trabalhadores em relação a essa exposição, em nosso país. Existem na literatura nacional apenas relatos e descrição de séries de casos $^{(7-10)}$.

Geralmente, a história ocupacional sugestiva é o critério mais prático e útil de avaliar a exposição ocupacional ao asbesto. Através de questionários estruturados, entrevistadores treinados poderão identificar pessoas com história ocupacional compatível com significativa exposição ao asbesto. Medidas ambientais podem ser usadas na estimativa dos niveis de fibras presentes nos locais de trabalho ou nos locais que utilizam o asbesto como matériaprima. O diagnóstico clínico de DRA baseia-se na entrevista detalhada do paciente quanto aos dados ocupacionais de exposição ao asbesto e latência, sinais e sintomas, radiologia, provas de função, exames citológicos seletivos, histopatologia e outros estudos laboratoriais ${ }^{(6)}$. Confirmação histopatológica é requerida para casos suspeitos de malignidade associada ao asbesto e para a resolução do diagnóstico diferencial.

Radiografia de tórax constitui-se na ferramenta básica de screening para identificar DRA, tais como asbestose, anormalidades pleurais, câncer de pulmão e mesotelioma. Também é largamente aceita a limitação da radiografia de tórax na detecção da asbestose e anormalidades pleurais associadas ao asbesto(6). Tomografia computadorizada (TC) e tomografia computadorizada de alta resolução (TCAR) podem facilitar a detecção de asbestose e alterações pleurais e malignidades associadas ao asbesto, não sendo, porém, ainda recomendadas como ferramentas de uso primário na investigação. 
Na grande maioria dos casos, a utilização de biópsias pulmonares em trabalhadores com história de exposição e de exames de imagem compativeis com asbestose ou placas pleurais não se faz necessária. Porém, já nos defrontamos com inúmeras outras situações em que o diagnóstico de outras doenças intersticiais em trabalhadores expostos ao asbesto foram evidenciadas somente pela histopatologia. Chamamos a atenção para, em caso de dúvidas inerentes à história ocupacional ou alterações de imagem pouco convincentes, dever esclarecer o diagnóstico de eventuais outras doenças que possam aparecer concomitante ou não com a asbestose (doenças fúngicas, tuberculose, doenças de pequenas vias aéreas, pneumonias intersticiais e outras causas). Durante nosso estudo, deparamo-nos com trabalhadores com alta carga de exposição ao asbesto (período compreendido entre 1940 e 1980) que apresentaram radiografia de tórax e TCAR normais. Outros trabalhadores, com pouco tempo de exposição (menor do que três meses) durante as décadas de 40, 50 e 60, apresentaram radiografia de tórax normal, porém a TCAR evidenciou a presença de placas pleurais. Estas TCAR evidenciaram, ainda, além das placas pleurais, nódulos espiculados, isolados, com aumentos de densidade pós-contraste maiores que 15 unidades Hosenfield e positivos quanto ao PET scan, sugerindo a necessidade de outros procedimentos diagnósticos mais invasivos. Com a indicação da biópsia ou outros procedimentos invasivos, caberá ao patologista a palavra final sobre a existência ou não de padrões histopatológicos característicos das DRA. Tais padrões histopatológicos não são de conhecimento universal, principalmente para o patologista não especialista em pulmão. Dessa forma, o amplo conhecimento dos padrões básicos de reações pulmonares, que na maioria das vezes são estereotipadas, constitui-se no primeiro passo para o diagnóstico correto. Essas circunstâncias específicas justificam a afirmativa de que as DRA são ainda consideradas de grande complexidade diagnóstica, necessitando de correlação entre a clínica, a imagem e a histopatologia.

Análise do tecido pulmonar para detecção de fibras e corpos de asbesto (CA) pode complementar a história ocupacional. Para propósitos clínicos, as seguintes contagens identificam pacientes com exposições elevadas ${ }^{(6)}$ :

- > 0,1 milhão de anfibólios (> 5 $\mu \mathrm{m}) /$ grama de pulmão seco ou um milhão de anfibólios (> 1 $\mu \mathrm{m})$ /grama de pulmão seco (microscopia eletrônica);

- > $1.000 \mathrm{CA} /$ grama de tecido seco $(100 \mathrm{CA} /$ grama tecido úmido) ou $>$ um corpo de asbesto/milímetro de lavado broncoalveolar (microscopia óptica).

Cada laboratório deverá estabelecer seus valores de referência, de acordo também com os niveis da população geral exposta.

\section{OBjetivos}

Ao longo da experiência que vimos desenvolvendo através de um estudo interinstitucional sobre a morbidade $e$ mortalidade entre trabalhadores expostos ao asbesto, o objetivo traçado para esta revisão é discutir critérios diagnósticos que envolvam o curso da história natural destas doenças e que terão importância para o diagnóstico final.

\section{ClassifiCAÇÃO DAS DOENÇAS RELACIONADAS AO ASBESTO}

No Quadro 1 estão relacionadas as principais doenças e sua associação com asbesto(11).

\section{CORPOS DE ASBESTO (CA) E CORPOS}

\section{FERRUGINOSOS}

O termo corpo ferruginoso refere-se a uma partícula mineral à qual macrófagos pulmonares adicionaram um envoltório de proteína férrica (ferritina ou hemossiderina). Dependendo da natureza do core, podem ser identificados vários tipos de corpos ferruginosos. Assim, os CA compõem um tipo de corpo ferruginoso cujo core é a fibra de asbesto. Dessa forma, corpos ferruginosos não são específicos para asbesto. Quando o core é outro, como, por exemplo, o talco, então temos o corpo de talco ou corpo ferruginoso à base de talco. Outros minerais que podem formar o core do corpo ferruginoso são: mica, carbono, carvão e fibras elásticas degradadas em hemorragias pulmonares crônicas.

A não ser em condições de alta exposição, crisotila em geral não forma $\mathrm{CA}$, que, quando visualizados, mais provavelmente representam os anfibólios.

CA podem ser identificados tanto em pessoas com exposição como na população em geral.

\begin{tabular}{|c|c|}
\hline \multicolumn{2}{|c|}{$\begin{array}{c}\text { QUADRO } 1 \\
\text { Doenças relacionadas ao asbesto }^{(11)}\end{array}$} \\
\hline Doença & Associação \\
\hline $\begin{array}{l}\text { Alterações pleurais não neoplásicas } \\
\text { - derrame pleural } \\
\text { - espessamento pleural difuso } \\
\text { - placa pleural } \\
\text { - atelectasia redonda }\end{array}$ & + \\
\hline Asbestose (fibrose intersticial) & + \\
\hline Câncer de pulmão & + \\
\hline Mesotelioma maligno (pleura, peritônio) & + \\
\hline
\end{tabular}




\section{ANÁlise DAS FIBRAS E CORPOS DE ASBESTO NOS TECIDOS HUMANOS}

Asbesto ocorre no pulmão em duas formas. A maioria do mineral encontra-se em forma de fibras não-revestidas, que é a na qual ele é inalado. No pulmão, uma minoria de fibras adquire um envoltório por proteína férrica, sendo então chamada de corpo de asbesto, uma forma específica de corpo ferruginoso ${ }^{(11)}$.

Uma variedade de técnicas pode permitir a avaliação qualitativa e quantitativa da presença de asbesto no tecido pulmonar ${ }^{(12)}$. Alguns pontos que devem ser considerados:

1) exames macroscópicos e microscópicos de rotina associados com dados radiológicos e clínicos apropriados são os métodos primários de diagnóstico das DRA ${ }^{(11)}$;

2) independente do número de corpos ou fibras encontrados, sua presença por si só não significa doença. A quantificação de fibras no tecido fornece estimativa do nível de exposição, que pode ou não ser indicador de determinada doença. Por exemplo, nível elevado de fibras de amosita ou contagem elevada de $\mathrm{CA}$ em paciente com mesotelioma usualmente implicará o asbesto como agente envolvido, mas o mesmo não poderá ser dito com certeza se for um carcinoma de pulmão(11);

3) com o uso de agentes digestivos no tecido, a quantificação será necessária e deverá ser comparada em relação à população geral|(12). Uma vez que a população tem asbesto nos pulmões, a mera demonstração qualitativa da presença de fibras ou CA em um tecido digerido não tem valor.

\section{- Métodos específicos de análise}

Técnicas como microscopia de polarização, coloração por dispersão e difração quantitativa por raios X são adequadas para identificar o asbesto de produtos específicos ou no ar, mas não podem ser usadas no tecido(11). Como regra, fibras retidas no corpo por algum tempo não são birrefringentes. Fibras não revestidas podem ser visiveis ao microscópio óptico; contudo, podem estar abaixo do poder de resolução do microscópio; e mesmo quando identificadas, não há como diferenciá-las de outros tipos de fibras comuns nos pulmões ${ }^{(11)}$.

Corpos de asbesto, por outro lado, são facilmente visíveis ao microscópio óptico, sendo marcadores específicos de asbesto na maioria da população(11). O método mais simples, rápido e menos dispendioso, mas também menos quantificável de determinar asbesto no pulmão, é o seu achado ou contagem do número de CA nos cortes histológicos. Pelo pequeno volume de tecido contido num corte histológico de rotina, em geral $5 \mu \mathrm{m}$, os preparados histológicos são muito insensiveis detectores de CA. Todavia, adotamos o mesmo critério proposto por Roggli et al. ${ }^{(13)}$, ou seja, conhecido o nível de exposição ao asbesto da população em questão (não devem ser encontrados após examinar de um a 100 cortes), a observação de um CA em um corte de $5 \mu \mathrm{m}$ usualmente indica exposição ocupacional. Para tanto, o patologista deverá rastrear a procura em 50 campos de grande aumento, utilizando para isso aprofundamento dos cortes. Como os CA não são facilmente visualizados nos cortes corados $\mathrm{H} \& \mathrm{E}$, colorações para ferro devem ser utilizadas, para facilitar ao patologista sua identificação. A contagem definitiva deverá ser efetuada por microscopia eletrônica após digestão enzimática dos cortes de pulmão. Essa técnica pode ser realizada mesmo após a inclusão do tecido pulmonar em blocos de parafina. Os índices numéricos finais deverão então ser comparados com índices da população exposta em geral e expressos através de alta ou baixa exposição(12). No Quadro 2 podem ser apreciados alguns valores.

Em nossa rotina diagnóstica, examinamos cortes de tecido pulmonar a $5 \mu \mathrm{m}$ corados pela H\&E e Perls, rastreando todos os campos possiveis se o espécimen for oriundo de biópsia transbrônquica, ou até 100 campos de grande aumento se a biópsia foi a céu aberto, aprofundando os cortes, se necessário. A seguir os blocos de parafina seguem para digestão enzimática $e$ análise sob o microscópio eletrônico.

\section{AlteraÇÕES PLEURAIS NÃO NEOPLÁSICAS}

A doença pleural é a manifestação mais importante de exposição ao asbesto ${ }^{(14)}$. As manifestações não malignas de exposição ao asbesto no espaço pleural incluem as placas pleurais circunscritas, o espessamento (fibrose) pleural difuso, atelectasia redonda e o derrame pleural relacionado ao asbesto.

\section{- Placas pleurais}

As placas pleurais, ao mesmo tempo em que são marcadores mais comuns de exposição ao asbesto ${ }^{(15)}$, consti-

\begin{tabular}{|c|c|c|c|c|}
\hline \multicolumn{5}{|c|}{$\begin{array}{c}\text { QUADRO } 2 \\
\text { Fibras de asbesto por microscopia eletrônica no pulmão }{ }^{(1,12)}\end{array}$} \\
\hline & Fibras/mg & Média/mg & Comprimento & Tipo \\
\hline População geral & $\begin{array}{l}<1.000 \\
<100\end{array}$ & 2 a 300 & $\begin{array}{l}<5 \mu \mathrm{m} \\
>5 \mu \mathrm{m}\end{array}$ & crisotila \\
\hline Minas (Quebec) & \multicolumn{4}{|c|}{10 vezes maior } \\
\hline Placas pleurais & \multicolumn{4}{|c|}{100 a $5.000 / \mathrm{mg}$} \\
\hline $\begin{array}{l}\text { Asbestose } \\
\text { - Grau } 0 \\
\text { - Grau I } \\
\text { - Grau II }\end{array}$ & \multicolumn{4}{|c|}{$\begin{array}{c}2.000 \text { a } 19.000 / \mathrm{mg} \\
135.000 / \mathrm{mg} \\
1.370 .000 / \mathrm{mg}\end{array}$} \\
\hline Mesotelioma & \multicolumn{4}{|c|}{ normal a asbestose } \\
\hline
\end{tabular}


tuem-se em DRA. Todas as fibras de asbesto têm igual capacidade de induzir placas pleurais, porém a extensão das placas não apresenta relação clara com exposição cumulativa. À macroscopia são lesões circunscritas, levemente sobrelevadas, esbranquiçadas, irregulares, encontradas na pleura parietal $e$, raramente, na pleura visceral $^{(11)}$. Podem ser pequenas ou extensas, comumente localizadas nos segmentos laterais e posterior das zonas médias dos pulmões, onde então acompanham o contorno das costelas e do diafragma. Comumente, elas acompanham as fissuras lobares e podem invadir o mediastino e pericárdio, raramente, porém, comprometendo os ápices ou sulco costofrênico. Histologicamente, placas pleurais associadas ao asbesto são caracterizadas por lesões pouco celulares, extensa colageinização através de fibras em característico arranjo em basket woven, delimitadas por fino revestimento de células mesoteliais. O envolvimento da pleura parietal é uniforme, com mínimo espessamento da pleura visceral, não havendo, porém, adesões entre os dois folhetos. Calcificações pleurais freqüentemente se desenvolvem sobre essas lesões fibro-hialinas ao longo do curso de exposição. A patogênese das placas pleurais é baseada no efeito direto das fibras que alcançam o espaço pleural ${ }^{(16)}$. Tendem a aumentar lentamente de tamanho durante décadas e raramente podem levar ao aparecimento do mesotelioma maligno difuso. São assintomáticas, desde que não estejam associadas a asbestose pulmonar ou obliteração do seio costofrênico, consistindo de achados incidentais à radiografia de tó$\operatorname{rax}^{(17)}$. Têm sido reconhecidas como causa de redução de valores nas provas de função pulmonar, principalmente a $\mathrm{CVF} / \mathrm{VEF}_{1}{ }^{(18)}$. Radiologicamente, podem ser visibilizadas ao longo da parede lateral, principalmente se calcificadas. As posições em oblíquo e a tomografia computadorizada (TC) aumentam a detecção. A TC é útil para detectar placas perivertebrais e pericárdicas, enquanto a TC de alta resolução clarifica as lesões diafragmáticas ${ }^{(19)}$. Usualmente, são bilaterais $(80 \%)$ e, quando unilaterais, podem ser devidas ao trauma, tuberculose prévia ou colagenoses. A histologia não é necessária para o diagnóstico na grande maioria dos casos.

\section{- Espessamento (fibrose) pleural difuso}

O espessamento (fibrose) pleural difuso associado ao asbesto pode ser localizado ou difuso, uni ou bilateral ${ }^{(14,20)}$. Macroscopicamente, as lesões variam de leve opacidade da superfície pleural a coloração brancacenta obliterando a transparência habitual da serosa. Espessamento difuso é freqüentemente visibilizado como uma tela de 5 a $10 \mathrm{~cm}$ de extensão craniocaudal, afetando em $90 \%$ dos casos o seio costofrênico, as fissuras interlobares e interlobulares. Enquanto as placas pleurais fibrosas comprometem a pleura parietal, o espessamento fibroso é uma doença da pleura visceral, que pode ocorrer mesmo em pacientes com mínima fibrose pulmonar ${ }^{(21)}$. Corpos ou fibras de asbesto são freqüentemente encontrados na pleura visceral, no parênquima subjacente, ou em ambos. Há três mecanismos diferentes envolvidos na fibrose pleural difu$\mathrm{sa}^{(20-22)}$. O primeiro é a confluência de grandes placas pleurais fibrosas ocorrendo em 10 a $20 \%$ dos casos. O segundo envolve a extensão da fibrose subpleural à pleura visceral em 10 a $30 \%$ dos casos. O mecanismo mais comum envolvido, no entanto, é a resolução fibrótica de derrame pleural, determinando, então, o espessamento. Esse mecanismo é altamente realçado pela constatação radiológica ou por toracocentese de derrame pleural prévio em um terço dos pacientes que desenvolveram fibrose pleural difusa. O mecanismo irritativo que desencadeará o processo fibrótico é idêntico ao das placas pleurais, diferindo apenas pela deposição das fibras nas áreas subpleurais parenquimatosas do pulmão.

A fibrose pleural difusa ocorre após curto tempo de intensa exposição ao asbesto, com tendência à progressão ao longo dos anos ${ }^{(22)}$. Quando leve, pode ser assintomática e descoberta como achado radiológico, enquanto a forma difusa da lesão freqüentemente conduz à dispnéia, tosse seca crônica e dor torácica, acompanhadas de disfunções respiratórias restritivas, que variam em função direta da resposta fibrótica ${ }^{21,22)}$. Raramente, porém, pacientes com doença bilateral grave desenvolvem insuficiência respiratória e morte. Na radiografia torácica, a fibrose pleural difusa apresenta-se como opacidade pleural contínua comprometendo acima de $25 \%$ da superfície pleural, freqüentemente envolvendo o ângulo costofrênico. Pode ser uni ou bilateral e associada à presença ou ausência concomitante de asbestose e calcificações pleurais ${ }^{(19)}$. Raramente, a fibrose pleural poderá determinar a formação de um pseudotumor com base pleural (atelectasia redonda). TC é particularmente útil em determinar as relações entre a fibrose pleural difusa e outras anormalidades pleurais, bem como diferenciar entre fibrose pleural e depósitos de gordura. A fibrose pleural difusa não é exclusiva da exposição ao asbesto e pode representar antiga reação inflamatória à tuberculose, cirurgia torácica, trauma torácico hemorrágico e reações a drogas. $\mathrm{O}$ padrão radiológico ajuda também na diferenciação, desde que o achado de alterações intersticiais bilaterais bibasais em associação com placas pleurais sugere fortemente o diagnóstico de exposição ao asbesto. A biópsia poderá ser requerida quando a lesão torácica for progressiva ou quando malignidade é o diferencial.

\section{- Atelectasia redonda}

Atelectasia redonda é uma rara complicação de doença pleural asbesto-induzida(23). É determinada por cicatrização $e$ fusão dos folhetos parietal e visceral, resultando 
na invaginação da pleura e aprisionamento do pulmão adjacente, conduzindo à atelectasia. O resultado é a formação de uma massa pseudotumoral, cuja TC demonstra continuidade entre as áreas de espessamento pleural difuso, evidências de perda de volume no pulmão adjacente, ou a característica "cauda de cometa" de vasos e brônquios entrando em uma massa bem delimitada. A TC demonstra ainda estabilidade da lesão ao longo dos anos, o que suporta um diagnóstico de lesão benigna, presença de placas pleurais ou alterações parenquimatosas, como evidências de exposição ao asbesto. A localização do processo envolve os segmentos inferior e posterior, podendo em um terço dos casos encontrar-se lesões múltiplas. Em outros, um derrame pleural lento pode preceder o aparecimento. Se a natureza benigna da lesão é duvidosa na análise radiológica, broncoscopia ou biópsia transparietal serão necessárias para afastar processo neoplásico.

\section{- Derrame pleural}

Derrame pleural agudo e benigno pode ser uma manifestação comum em pacientes com 30 a 40 anos de exposição ao asbesto ${ }^{(24)}$. O período de latência do derrame é mais curto que o das placas pleurais, mesotelioma maligno, ou câncer de pulmão, ocorrendo em geral nos primeiros 12 a 15 anos após início da exposição. Em 50\% dos pacientes, sintomas ocorrerão na vigência de pleurisia (dor torácica, dispnéia, tosse e febre), com derrame hemorrágico, exames bioquímicos normais, eosinofilia e presença de células mesoteliais. Raramente, corpos de asbesto (CA) são encontrados, embora eles possam estar presentes no tecido pulmonar subjacente. O derrame pode persistir por seis meses ou mais, sofrer reabsorção espontânea, ou recorrer contralateralmente, não sendo indicação de mesotelioma futuro, embora pacientes tenham risco de desenvolver asbestose tanto quanto os com fibrose pleural difusa. Adicionalmente, pacientes com derrame associado ao asbesto têm maior risco de desenvolver fibrose pleural difusa ${ }^{(1)}$. A biópsia pleural é necessária, principalmente, para afastar diagnóstico de mesotelioma maligno. Histologicamente, o quadro é dominado por pleurisia fibrosa crônica com mínima celularidade. Seguimento do paciente também é requerido, uma vez que o diagnóstico definitivo de derrame pleural benigno não poderá ser estabelecido até que seja constatado um intervalo de três anos livre de tumor ${ }^{(1)}$.

\section{AsBestose}

Asbestose é definida como uma fibrose intersticial difusa dos pulmões como conseqüência da exposição ao asbesto. As características clínicas tanto quanto histopatológicas da fibrose são semelhantes a outras causas de fibrose intersticial, excetuando-se o achado de fibras ou corpos de asbesto (CA) no tecido pulmonar, além das quan- tidades comumente encontradas na população em geral. Sintomas de asbestose incluem tosse e dispnéia e estertores basilares inspiratórios. É descrito baqueteamento dos dedos, porém estas alterações são raramente observadas em nosso meio. Alterações funcionais respiratórias podem ocorrer numa percentagem de casos e incluem anormalidades nas trocas gasosas, um padrão restritivo e obstrutivo devido à doença de pequenas vias aéreas ou mais comumente pelo fumo. Tabagismo atual ou no passado é detectado em $50-70 \%$ dos nossos pacientes. Nesse contexto, sua abordagem deverá ser multidisciplinar e incluir epidemiologia, clínica, radiologia, provas de função pulmonar e patologia. Na rotina diagnóstica, especialmente para o patologista geral, há dificuldades na caracterização morfológica da doença, sobretudo pela falta de critérios padronizados à microscopia óptica e da necessidade em realizar microscopia eletrônica para contagem e identificação das fibras. Nesse contexto, é problemática a questão da indicação da ME de rotina em algumas situações, pelo simples fato de praticamente não se disporem de centros especializados para fazê-la no país. Realmente, em nossa rotina diagnóstica é difícil realizar a ME, uma técnica bastante sofisticada, restrita na maioria das vezes a alguns centros especializados e, mesmo em tais centros, desprovida do equipamento necessário para a contagem das fibras. Seguramente, tais métodos não são primários e correspondem a exceções na prática médica. Contudo, apesar das dificuldades, entendemos que a contagem do tipo e número de fibras por grama de pulmão seco, bem como a contagem do número de $\mathrm{CA}$, devam ser praticados sempre que possivel, pois poderão fornecer informações específicas do tipo de exposição, especialmente a presença de anfibólios e sua comparação com as populações não expostas. Torna-se, portanto, imperioso o conhecimento dos padrões morfológicos de apresentação da doença pulmonar e, sobretudo, sua correlação com a radiologia.

Asbestose associada com altos níveis de exposição está geralmente também associada com sinais radiológicos de fibrose parenquimatosa. Todavia, é possivel que fibrose leve possa ocorrer a baixos niveis de exposição e critérios radiológicos nem sempre serão preenchidos em casos de fibrose parenquimatosa detectada histologicamente. O reconhecimento da asbestose à radiografia de tórax segue os padrões preconizados pela classificação ILO ${ }^{(19)}$, na qual achados radiológicos de pequenas opacidades são aceitos como estádios iniciais de asbestose. TCAR poderá confirmar os achados radiológicos de asbestose e detectar alterações iniciais não identificadas à radiografia de tórax, mas deverá ser realizada em casos selecionados. Os efeitos do fumo deverão ser considerados na avaliação clínica e funcional respiratória da asbestose precoce, provas de função e sintomas respiratórios. 
Do ponto de vista da anatomia patológica, por doença intersticial difusa entende-se o comprometimento inflamatório/fibrótico/remodelador do tecido conjuntivo de sustentação das estruturas parenquimatosas pulmonares, a saber, o interstício axial (vias aéreas), septal (alvéolos) e periférico (subpleural).

No Quadro 3 é apresentada a classificação para as formas histoanatômicas de envolvimento pulmonar na exposição ao asbesto. A fim de padronizar o diagnóstico histológico e a graduação da asbestose, em nossa rotina temos utilizado a classificação modificada do CAP-NIOSH ${ }^{(25)}$ e preconizada por Roggli et al. ${ }^{(13)}$, por ser mais abrangente e levar em consideração lesões precoces e avançadas.

Lesões precoces envolvem o interstício axial de sustentação das vias aéreas (bronquíolos terminais), sendo por isso classificadas por alguns autores como "bronquiolites associadas ao asbesto"(11) ou "fibrose intersticial grau I"(13) Subseqüentemente, a doença progride para envolver o ácino pulmonar, ou seja, o interstício de sustentação dos bronquíolos respiratórios, ductos e alvéolos adjacentes, sendo então classificada como "fibrose intersticial graus II, III e IV"(13) ou, respectivamente, fibrose intersticial graus II, III e pneumonia intersticial usual ${ }^{(11)}$, de acordo com a extensão histoanatômica de envolvimento. A fibrose intersticial grau IV ${ }^{(13)}$ ou asbestose pulmonar ${ }^{(11)}$ corresponde ao padrão histológico "pneumonia intersticial usual UIP”, da Classificação Internacional das Pneumonias Intersticiais Idiopáticas proposta recentemente pela ATS/ $\mathrm{ERS}^{(26)}$.

O quadro histopatológico é inicialmente dominado por reação inflamatória rica em células linfóides e macrofági- cas, que alargam e deformam o tecido conjuntivo intersticial, numa seqüência progressiva envolvendo bronquíolos e tecido alveolado do ácino pulmonar (ductos, sacos e alvéolos). A interação macrófago/linfócito culmina com a liberação de substâncias ativadas, citocinas, responsáveis pela digestão enzimática do citoesqueleto pulmonar $e$ ativação de fibroblastos, que dão início ao remodelamento pulmonar. Com o progredir da doença, instala-se a obliteração fibrótica e remodelamento do ácino pulmonar, com conseqüente faveolamento e perda funcional das áreas de trocas gasosas. As fibras de asbesto depositam-se ao longo das bifurcações.

A sistemática para o diagnóstico de asbestose pulmonar deverá incluir:

- I) biópsia pulmonar aberta

- II) histopatologia

- III) diagnóstico de asbestose pulmonar - correlação clínico-radiológico-funcional e grau de exposição

- IV) identificação e contagem de fibras e CA ao microscópio eletrônico

- V) dificuldades diagnósticas

\section{I) Biópsia pulmonar aberta}

É abordagem indicada, pois permite classificar o processo dentro dos compartimentos pulmonares. A biópsia transbrônquica, por vezes indicada em pacientes sem condições para abordagem invasiva, poderá trazer informações quanto ao estadiamento do processo inflamação/ fibrose e na eventual presença do CA, porém não permitirá determinar o padrão histopatológico de envolvimento pulmonar.

\begin{tabular}{|c|c|c|c|}
\hline \multicolumn{4}{|c|}{$\begin{array}{c}\text { QUADRO } 3 \\
\text { Classificação dos padrões histoanatômicos de envolvimento pulmonar na exposição ao asbesto }\end{array}$} \\
\hline & CAP-NIOSH ${ }^{(25)}$ & Churg e Green ${ }^{(11)}$ & Roggli et al. ${ }^{(13)}$ \\
\hline $\begin{array}{l}\text { - Interstício axial } \\
\text { (bronquíolos terminais) }\end{array}$ & $\begin{array}{l}\text { Fibrose intersticial } \\
\text { - Grau I }\end{array}$ & $\begin{array}{l}\text { - Bronquiolite } \\
\text { associada } \\
\text { ao asbesto }\end{array}$ & $\begin{array}{l}\text { Fibrose intersticial } \\
\text { - Grau I }\end{array}$ \\
\hline $\begin{array}{l}\text { - Interstício axial + } \\
\text { (bronquíolos respiratórios) } \\
\text { - Interstício septal } \\
\text { - Interstício periférico } \\
\quad= \\
\text { (ácino pulmonar) }\end{array}$ & $\begin{array}{l}\text { Fibrose intersticial } \\
\text { - Grau II } \\
\text { - Grau III ou } \\
\text { - Grau IV }\end{array}$ & $\begin{array}{l}\text { - Asbestose } \\
\text { pulmonar } \\
\text { (pneumonia } \\
\text { intersticial } \\
\text { usual (UIP)) }\end{array}$ & $\begin{array}{l}\text { Fibrose intersticial } \\
\text { - Grau II } \\
\text { - Grau III } \\
\text { - Pneumonia } \\
\text { intersticial } \\
\text { usual (UIP) }\end{array}$ \\
\hline
\end{tabular}




\section{II) Histopatologia}

Os critérios histopatológicos incluem:

- localização histoanatômica do processo no interstício pulmonar;

- extensão do processo nos compartimentos pulmonares;

- determinação da injúria parenquimatosa;

- determinação da reação parenquimatosa à injúria;

- determinação do padrão histológico de envolvimento fibrose intersticial grau I

fibrose intersticial graus II e III

pneumonia intersticial usual ou fibrose pulmonar grau IV

- identificação do CA

$\mathrm{H} \& \mathrm{E}$

Perls

- classificação do padrão histoanatômico associado ao asbesto (Quadro 3)

O primeiro critério histológico de envolvimento pulmonar será localizar, ao menor aumento do microscópio, o processo no interstício pulmonar: axial, septal ou periférico. A seguir, ainda no menor aumento, determina-se a extensão do processo nos compartimentos pulmonares: lobular (bronquíolo terminal) e acinar (bronquíolo respiratório, ductos e alvéolos). Passa-se, então, a determinar, agora ao maior aumento do microscópio, o tipo de injúria (necrose, degeneração, edema, hemorragia, infarto, neoplasia, etc.) e reação (inflamação/reparação/remodelamento-cistos). Nessa fase já será possível determinar o padrão histológico geral de envolvimento pulmonar em três entidades: fibrose intersticial grau I, fibrose intersticial grau II/III, pneumonia intersticial usual ou fibrose intersticial grau IV.

- fibrose intersticial grau I: inflamação/fibrose envolvendo o interstício axial peribronquíolo terminal com ou sem extensão ao interstício septal dos alvéolos imediatamente adjacentes ao lóbulo pulmonar;

- fibrose intersticial grau II ou III: inflamação/fibrose envolvendo o interstício axial, com extensão ao interstício septal dos alvéolos do ácino;

- pneumonia intersticial usual: áreas de inflamação/ fibrose/cistos, envolvendo o interstício axial, septal e periférico do ácino, em uma caraterística heterogeneidade temporal ao menor aumento do microscópico, intercaladas com áreas de pulmão normal.

O próximo critério deverá incluir a identificação de CA, inicialmente à $\mathrm{H} \& \mathrm{E} e$, posteriormente, sob coloração com o azul da Prússia (técnica do Perls). A procura pelos CA deverá incluir o rastreamento de um a 100 campos de grande aumento, utilizando cortes seriados do espécimen ${ }^{(27)}$. A identificação de um corpo de asbesto na vigência de um dos três padrões histológicos descritos fecha o diagnóstico de fibrose intersticial ou pneumonia intersti- cial usual associada ao asbesto. A classificação da fibrose intersticial ao asbesto deverá ser graduada de acordo com a extensão de comprometimento do lóbulo ou ácino pulmonar (Quadro 3).

\section{III) Diagnóstico final de asbestose pulmonar}

O diagnóstico histológico final de asbestose incluirá a identificação de fibrose intersticial difusa em tecido pulmonar adequadamente inflado, em locais distantes à presença de um câncer ou outras tumorações, acrescido da presença de dois ou mais CA em cortes de tecido de $1 \mathrm{~cm}^{2(12,27)}$ ou contagem de fibras não revestidas além dos padrões determinados pelo laboratório em questão(6).

\section{IV) Contagem de fibras ao microscópio eletrônico}

$\mathrm{Na}$ impossibilidade de ser efetuada de rotina, seu uso é obrigatório para o diagnóstico definitivo de asbestose pulmonar em duas situações. Na primeira, quando houver história de alta exposição durante o período crítico, curso clínico de doença intersticial difusa, porém com padrão histopatológico incaracterístico (por exemplo, uma granulomatose ou BOOP). Na segunda, há o padrão histopatológico característico, sem a presença do corpo de asbesto, curso clínico favorável, porém a história é de baixa exposição ocupacional ou fora do período crítico.

\section{V) Dificuldades para o diagnóstico}

Há evidencias de que casos raros de asbestose ocorrem sem significante número de CA. Esses casos são reconhecidos - distinguíveis da fibrose pulmonar idiopática - somente pela análise de fibras não revestidas em pulmão seco. Alguns casos de asbestose relacionada à exposição de crisotila pura podem ocorrer, com prolongado intervalo entre a última exposição e o diagnóstico e poucos ou não detectáveis $\mathrm{CA}$, além de baixa contagem de fibras no pulmão seco. A existência de tais casos é especulativa e, se o diagnóstico puder ser feito, deverá ser realizado em outras bases clínicas ou radiológicas, combinadas aos dados de exposição.

Padrões fibro-inflamatórios outros além da convencional asbestose têm sido descritos para trabalhadores com exposição ocupacional ao asbesto, incluindo um padrão semelhante ao da pneumonia intersticial descamativa (DIP), a ocorrência de inflamação granulomatosa, um quadro que lembra pneumonia intersticial linfóide, e bronquiolite obliterante com pneumonia organizante. Embora o padrão DIP com CA seja provavelmente asbesto-relacionado, os outros padrões não têm sido associados.

As dificuldades maiores para o diagnóstico final de asbestose incluirão algumas situações citadas a seguir. $\mathrm{Na}$ ausência de um dos três padrões histopatológicos descritos, porém com o corpo de asbesto presente, o diagnóstico final de asbestose deverá embasar-se na história ocu- 
pacional, no curso clínico-radiológico-funcional de doença pulmonar difusa e, mais importante, na identificação $e$ contagem de fibras pela microscopia eletrônica. Só nessas situações é que, por exemplo, um padrão histopatológico de pneumonia organizante (antiga BOOP) ou sarcoidose poderá ser a forma de apresentação da asbestose pulmonar. Em outra situação, como, por exemplo, diante de um padrão histopatológico de UIP com ausência de corpo de asbesto, de curso clínico-radiológico-funcional para doença pulmonar difusa, porém com contagem de fibras inferior ou dentro dos padrões encontrados na população geral, o diagnóstico de asbestose pulmonar deve ser cauteloso, mesmo na vigência de história ocupacional compativel. Nesse caso, outras causas (colagenoses, idiopática) deverão ser investigadas como base para a UIP. Da mesma forma, diante de um padrão histopatológico de UIP sem o CA, com impossibilidade de realizar microscopia eletrônica, porém com história de alta exposição ocupacional, seguindo curso clínico-radiológico-funcional de doença pulmonar difusa, será potencialmente viável estabelecer o diagnóstico de asbestose pulmonar.

Pelo que foi exposto, depreende-se que o patologista, clínico e radiologista devem estar atentos para a presença obrigatória dos seguintes requisitos antes de estabelecer um diagnóstico de asbestose pulmonar:

- padrão histopatológico de comprometimento inflamatório/fibrótico do interstício pulmonar, na forma de fibrose intersticial (graus II, III e IV);

- exposição ocupacional;

- curso clínico-radiológico-funcional de doença pulmonar difusa;

- contagem e determinação de fibras à microscopia eletrônica acima dos níveis padronizados para a população em geral.

\section{CÂNCER DE PULMÃo}

O risco de câncer de pulmão entre populações expostas ao asbesto é sabidamente conhecido. Entretanto, sua ocorrência depende, entre outros, da carga ou dose de exposição e do tipo de fibra. Inúmeros estudos epidemiológicos demonstram essa correlação(28-30). Tal risco é maior ainda quando a asbestose está presente $e^{(30-32)}$; o risco relativo também parece estar aumentado em relação à gravidade da fibrose pulmonar e da carga de fibras no pulmão(33). Alguns estudos revelam altas freqüências, acima de $40 \%$, de câncer de pulmão em portadores de asbesto$\mathrm{se}^{(34,35)}$, enquanto outras pesquisas mostram proporções de até $18 \%$ em algumas coortes(36).

Os quatro tipos maiores de câncer de pulmão (escamoso, adenocarcinoma, pequenas e grandes células) podem estar relacionados com a exposição ao asbesto, sendo possivel a ocorrência de qualquer um deles, não havendo predileção para um tipo histológico ou outro, e não diferindo daqueles atribuídos a outras causas ${ }^{(31)}$.

Em qualquer circunstância é de fundamental importância estimar a carga ou dose de exposição, pois, com pouco tempo de exposição em altas concentrações, o risco para seu aparecimento é de duas ou mais vezes ${ }^{(6)}$. Em exposições muito elevadas (sinais de asbesto na atividade ocupacional de isolamento térmico ou acústico), o risco de câncer de pulmão pode dobrar, mesmo com exposições menores que um ano.

O risco relativo para esse tipo de tumor é estimado de 0,5 a $4 \%$ para cada fibra por centímetro cúbico por ano (fibra/ano) da exposição cumulativa, que ao nível de 25 fibras/ano tem risco estimado duas vezes maior de ocorrência desse tumor. A carga pulmonar de dois milhões de fibras de anfibólio maior que $5 \mu \mathrm{m}$ por grama de pulmão seco ou de 5 milhões de fibras de anfibólio menor que $1 \mu \mathrm{m}$ pode correlacionar-se com o dobro do risco de aparecimento do câncer de pulmão(6) (esta é seguramente uma das principais razões para que, sempre que possivel, deva ser determinada a carga de tipos de fibras no tecido pulmonar, bem como a contagem de CA no tecido e lavado broncoalveolar).

Essa concentração de fibras guarda um paralelismo com a contagem de CA. O achado de 5.000 a 15.000 CA por grama de pulmão seco ou de 5 a $10 \mathrm{CA}$ por ml de LBA é comparável com carga de fibras; quando essa concentração é menor que $10.000 \mathrm{cA} /$ grama de pulmão seco, a análise de fibras por ME é recomendada ${ }^{(6)}$.

Sabendo-se que a crisotila, em função de seu clearance mais rápido ou menor biopersistência não se acumula nos pulmões, da mesma forma que os anfibólios, considera-se como melhor indicador para risco de câncer de pulmão a exposição cumulativa em fibras/ano e não a análise da carga de fibras no tecido pulmonar.

Devido à alta incidência de câncer de pulmão na população geral, fumantes e não fumantes (30\% ocorrem em não fumantes e não expostos ao amianto) ${ }^{(31)}$, não é possivel provar com precisão determinística que o asbesto seja o fator causal para determinado paciente, visto como um indivíduo, mesmo quando a asbestose está presente. As estimativas do risco relativo de câncer de pulmão relacionado com a exposição ao asbesto são baseadas em diferentes estudos de bases populacionais nas quais não se evidencia consenso, mas, sim, pontos controversos. Entretanto, elementos vinculados a causas requerem conclusões médicas baseadas em análises probabilísticas para que se possa inferir ou imputar a essa exposição o fator causal ou contribuinte para a doença. A exposição cumulativa ao asbesto, em bases probabilísticas, deve ser considerada como um dos principais critérios para atribuição do risco para câncer de pulmão. 
A presença de asbestose é um indicador de alta exposição e também pode ser considerada como risco adicional para o câncer de pulmão. Entretanto, alguns autores referem que a não ocorrência de asbestose não seja condição para excluir essa fibra como fator contribuinte para o aparecimento da neoplasia ${ }^{(6,31)}$. No mesmo sentido, a presença de placas pleurais é um indicador de exposição ao asbesto. Quando isoladas, ou pouco expressivas, podem ser correlacionadas com baixas exposições. Quando muito expressivas, bilaterais, extensas, com espessamento pleural difuso, bilaterais ou não, podem ser associadas a exposições mais intensas. Esses casos devem ser considerados como preditivos ou indicadores de morbidade e sua atribuição ao câncer deverá ter suporte consistente na história ocupacional, com substancial exposição cumulativa e contagem de fibras e CA no tecido pulmonar(12,29). Dessa forma, consideramos que em estudos epidemiológicos prospectivos os portadores de placas pleurais deverão ser reavaliados, tendo em vista esses fatores que atribuem maior risco de neoplasia.

Embora os efeitos do consumo tabágico nos casos de câncer de pulmão sejam sabidamente conhecidos e estimados, os efeitos do risco de exposição ao asbesto devem ser devidamente considerados, especialmente conhecendo-se suas ações sinérgicas multiplicativas ${ }^{(6)}$.

\section{MESOTELIOMA MALIGNO}

Mesotelioma maligno compromete qualquer membrana serosa e pode ser induzido por inalação do asbesto(37,38). Estudos epidemiológicos e análises mineralógicas cuidadosos estabelecem diretas evidências de que a maioria dos mesoteliomas é encontrada em indivíduos expostos ao amianto ou asbesto(27). Mesmo em casos de mesoteliomas nas mulheres, que são de duas a dez vezes menos comuns que nos homens, análise mineralógica dos tecidos para detecção de fibras minerais revela contagens de anfibólios nos pulmões maior do que nos controles ${ }^{(11)}$.

Com exceção de certos tipos histológicos de mesotelioma que são benignos, ou de potencial intermediário (e.g. : mesotelioma multicístico, mesotelioma benigno papilífero), todos os tipos de mesotelioma maligno podem ser induzidos pelo asbesto, com os anfibólios mostrando maior potencial carcinogênico do que a crisotila ${ }^{(38,39)}$.

Estudos em animais demonstram que o processo tem início com uma reação granulomatosa após injeção das fibras na pleura, subseqüentemente colageinizando, coalescendo e circunscrita por uma camada de células mesenquimais e células mesoteliais( ${ }^{(40)}$. Segue-se a transformação neoplásica com extensa substituição da superfície por células mesenquimais e epiteliais. Todas as fibras de asbesto têm potencial para produzir mesotelioma. $\mathrm{O}$ asbesto age como iniciador e promotor, sendo por isso con- siderado um carcinógeno completo, pelo menos na indução do mesotelioma em animais. A grande propensão de os anfibólios induzirem mais mesoteliomas no homem está relacionada às suas propriedades geométricas (fibras longas e finas), favorecendo sua penetração profunda e resistência à degradação, o que, em termos gerais, favorece sua persistência nos tecidos. Ainda não se estabeleceu definitivamente se as fibras que iniciam o mesotelioma necessitam de translocação para o espaço pleural ou podem agir a partir do parênquima pulmonar subpleural em contato direto com as células mesoteliais ou via rede de citocinas $^{(40)}$.

Contagens de fibras nos pulmões excedendo os padrões estabelecidos pelo laboratório em questão ou a presença radiológica ou patológica de lesão tecidual associada ao asbesto (e.g.: asbestose ou placas pleurais) ou evidências histopatológicas de conteúdo anormal de asbesto (e.g.: CA nos cortes histológicos de pulmão) deverão ser suficientes para relacionar um caso de mesotelioma à exposição ao asbesto em bases de probabilidade. Na ausência de tais marcadores, história de significante exposição ocupacional, doméstica ou ambiental é suficiente ${ }^{(6)}$. Há evidências de que mesoteliomas peritoneais estão associados com maiores niveis de exposição ao asbesto do que os mesoteliomas pleurais ${ }^{(41)}$. Questões duvidosas colocamse em casos de mesotelioma para os quais a contagem de fibra em pulmão ou pleura secos se situa dentro dos padrões preconizados para a população geral.

Os seguintes pontos deverão ser considerados ao estabelecer a etiologia ocupacional(6):

- A grande maioria dos mesoteliomas é devida à exposição ao asbesto.

- Mesotelioma pode ocorrer em casos com baixa exposição ao asbesto. Todavia, cargas baixíssimas de exposição traduzem riscos extremamente baixos.

- Em torno de $80 \%$ dos pacientes com mesotelioma referem algum grau de exposição ocupacional ao asbesto, de forma que sempre deverá ser investigada cuidadosamente a história ocupacional e ambiental.

- Uma história ocupacional de breve exposição deverá ser considerada como suficiente para que o mesotelioma seja designado como relacionado à ocupação.

- Um mínimo de dez anos a partir da primeira exposição é requerido para atribuir o mesotelioma à exposição ao asbesto, mesmo que na maioria dos casos o intervalo de latência seja maior (30 a 40 anos).

O fumo não tem influência no risco para o mesotelioma.

Marcadores histológicos, imuno-histoquímicos e ultraestruturais para o diagnóstico são bem estabelecidos(38). Consultas a especialistas devem ser realizadas em casos atípicos, ou naqueles nos quais o diagnóstico é incerto devido às discordâncias dos achados ou nos quais a quan- 
tidade de material disponivel é insuficiente para o diagnóstico definitivo. Mesoteliomas freqüentemente apresentam-se com derrame pleural, dispnéia e dor torácica ${ }^{(42)}$

\section{Dificuldades no diagnóstico dos mesoteliomas malignos}

O diagnóstico do mesotelioma maligno apresenta dois problemas maiores. O primeiro diz respeito ao estabelecimento do diagnóstico com base nas evidências patológicas. O segundo é diferenciar mesoteliomas de carcinomas e sarcomas metastáticos à pleura ou peritônio e de sarcomas primários da parede torácica. Todos esses tumores podem mimetizar intimamente a apresentação macroscópica e microscópica do mesotelioma ${ }^{(1,11,31,38)}$. Separação de um tumor primário localizado de pleura pode ocasionalmente ser um problema diagnóstico. Embora a literatura mais antiga sugira que não é possivel firmar o diagnóstico de mesotelioma sem autópsia, a maioria dos mesoteliomas, assim como outros tumores humanos, mostram características macro e microscópicas, assim como padrões histoquímicos e imuno-histoquímicos, de forma que biópsias cirúrgicas abertas ou grandes biópsias toracoscópicas mostram-se adequadas para os propósitos finais de diagnóstico(38). Biópsias por agulha algumas vezes permitem o diagnóstico, mas menos freqüentemente que biópsias abertas. Já o diagnóstico do mesotelioma em bases citológicas permanece controverso.

O Quadro 4 mostra um esquema para subclassificar mesoteliomas malignos por exame histológico ${ }^{(38,42)}$. $\mathrm{Ge}-$ nericamente, podem ser divididos em epiteliais (75\%), sarcomatosos (22\%) e mistos (24\%).

Colorações para mucina podem ser muito úteis para separar mesoteliomas de carcinomas metastáticos à pleura (Quadro 5). Por definição, tumores produtores de mucina PAS-positivo são adenocarcinomas. Mesoteliomas produzem ácido hialurônico que se cora por Alcian Blue ou ferro coloidal e pode ser removido por pré-digestão com

\begin{tabular}{|c|}
\hline $\begin{array}{c}\text { QUADRO } 4 \\
\text { Padrões histológicos do mesotelioma maligno }^{(11,38)}\end{array}$ \\
\hline $\begin{array}{l}\text { Epitelial } \\
\text { - tubulopapilífero } \\
\text { - epitelióide (células mesoteliais-símiles) } \\
\text { - adenomatóide } \\
\text { - desmoplásico }\end{array}$ \\
\hline $\begin{array}{l}\text { Sarcomatoso } \\
\text { - semelhante a fibrossarcoma ou fibro-histiocitoma maligno } \\
\text { - com elementos heterólogos lembrando condrossarcoma ou os- } \\
\text { teossarcoma desmoplásico }\end{array}$ \\
\hline Misto epitelial e sarcomatoso \\
\hline
\end{tabular}

hialuronidase. Colorações para mucina podem ser positivas em até $50 \%$ de casos histologicamente aceitáveis como mesoteliomas, o que torna a sensibilidade da imuno-histoquímica maior que a mucina para o diagnóstico.

O consenso na literatura para o painel imuno-histoquímico a ser utilizado para separar mesoteliomas e carcinomas é apresentado no Quadro 6. Os marcadores mais usados para carcinomas são CEA, B72.3, Leu M1, BER-EP4 combinados, conferindo boa especificidade $e^{(11,38)}$. Outros marcadores são mostrados no Quadro 6, mas sua eficácia é duvidosa. Torna-se crucial lembrar que mesoteliomas ocasionais corarão por marcadores de carcinomas, de maneira fraca, e alguns carcinomas também o farão

\begin{tabular}{|lcl|}
\hline \multicolumn{3}{|c|}{$\begin{array}{c}\text { QUADRO 5 } \\
\text { Colorações histoquímicas para mesoteliomas e carcinomas }\end{array}$} \\
\hline \multicolumn{1}{|c|}{ Coloração } & Mesotelioma & Adenocarcinoma \\
\hline $\begin{array}{l}\text { Ferro coloidal ou } \\
\text { Alcian Blue }\end{array}$ & Positivo & $\begin{array}{l}\text { Positivo } \\
\text { ou negativo }\end{array}$ \\
\hline $\begin{array}{l}\text { Ferro coloidal ou } \\
\text { Alcian Blue pré-digerido } \\
\text { com hialuronidase }\end{array}$ & Negativo & $\begin{array}{l}\text { Positivo antes } \\
\text { da digestão, } \\
\text { permanece } \\
\text { positivo }\end{array}$ \\
\hline $\begin{array}{l}\text { PAS (diástase-resistente) } \\
\text { ou mucicarmim }\end{array}$ & Negativo & Positivo \\
\hline
\end{tabular}

\begin{tabular}{|c|c|c|}
\hline \multicolumn{3}{|c|}{$\begin{array}{c}\text { QUADRO 6 } \\
\text { Imuno-histoquímica para carcinomas e mesoteliomas }{ }^{(11,38)}\end{array}$} \\
\hline Antígeno & Mesotelioma & Carcinoma \\
\hline Queratina & + & + \\
\hline Vimentina & + & + \\
\hline EMA & + & + \\
\hline CEA & - & + \\
\hline Leu M1 & - & + \\
\hline B72.3 & - & + \\
\hline HMFG & $+^{*}$ & $+^{*}$ \\
\hline BER-EP4 & \pm & + \\
\hline Componente secretório & - & + \\
\hline Lactogênio placentário humano & - & + \\
\hline Antígenos grupo sanguíneo Lewis & - & + \\
\hline Trombomodulina & + & + \\
\hline K1 & + & - \\
\hline ME1 & + & + \\
\hline HBME1 & $+^{*}$ & $+^{*}$ \\
\hline Calretinina & + & - \\
\hline \multicolumn{3}{|c|}{$\begin{array}{l}\text { Principais reações. A maioria dos anticorpos que cora primariamente carcinomas também } \\
\text { cora ocasionalmente mesoteliomas e vice-versa. } \\
\text { * Coloração citoplasmática vista somente em carcinomas; forte coloração da membrana } \\
\text { citoplasmática vista só nos mesoteliomas. }\end{array}$} \\
\hline
\end{tabular}


para marcadores de mesoteliomas. Um critério estatístico útil é que duas ou mais reações positivas aumentarão de 75 a 95\% a probabilidade a favor de carcinoma.

Tanto mesoteliomas como carcinomas coram para o antígeno de membrana epitelial - EMA, também não separando entre proliferações mesoteliais benignas e malignas. A observação de que mesoteliomas são positivos para queratina e vimentina perdeu força, porque boa proporção de carcinomas pode expressar os dois marcadores. Queratina é muito útil para separar mesoteliomas sarcomatosos de sarcomas.

K1 pode ser específico para mesoteliomas, mas só funciona em cortes por congelamento, da mesma forma que $\mathrm{ME}$, que também cora carcinomas.

HBME1 funciona em parafina, mas cora $50 \%$ dos adenocarcinomas. Trombomodulina cora mesoteliomas $e$ poucos carcinomas, mas é freqüente em adenocarcinomas.

A calretinina cora $100 \%$ dos mesoteliomas e apenas $10 \%$ dos carcinomas em cortes de parafina. Não há uma reação imuno-histoquímica definitiva para o diagnóstico e seu uso deve sempre ser valorizado à luz da apresentação histológica e macroscópica da lesão.

A maioria das características ultra-estruturais dos mesoteliomas é inespecífica, refletindo diferenciação de células epiteliais secretoras ou células mesenquimais fibroblásticas e variantes intermediárias e/ou pouco diferenciadas $^{(11)}$. Microvilos estão presentes na superfície das for-

\section{REFERÊNCIAS}

1. Begin R, Samet JM, Shaikh RA. Asbestos. In: Harder P, Schenker $\mathrm{MB}$, Balmes SR, eds. Occupational and environmental respiratory disease 1996;20:293-321.

2. Murray R. Asbestos: a chronology of its origins and health effects. $\mathrm{Br} \mathrm{J}$ Ind Med 1990;47:361-365.

3. Selikoff IJ, Lee DHK. Asbestos and diseases. New York: Academic Press, 1978.

4. De Capitani EM. Alterações pulmonares e pleurais causadas pela exposição ao asbesto: uma revisão. J Pneumol 1994;20:207-218.

5. Browne K. Asbestos related disorders. In: Parkers RW, ed. Occupational lung disorders. $3^{\text {rd }}$ ed. Oxford London: Butterworth-Heinemann, 1994;441-504.

6. Consensus Report. Scand J Work Environ Health 1997;23:311-316.

7. Algranti E. As doenças ligadas à exposição ao asbesto. Rev Bras Saúde Ocup 1986;14:15-16.

8. Algranti E. Riscos à saúde causados pelo asbesto e o controle médico. Rev Bras Saúde Ocup 1988;16:10-11.

9. Algranti E, Lima CQ, Vieira AV. Asbesto e carcinoma broncogênico: pesquisa de fibras em tecido pulmonar de três pacientes portadores de carcinoma broncogênico. Rev Paul Med 1989;107:133-138.

10. Amâncio JB, Bonciani M, Urquiza SD. Avaliação radiológica de trabalhadores da indústria de fibrocimento do Estado de São Paulo. Rev Bras Saúde Ocup 1988;16:51-55.

11. Churg A, Green FHY. Pathology of occupational lung disease. $1^{\text {st }} e d$. New York: 1989, Igaku-Shoin. mas epiteliais e por vezes também das formas fibroblásticas. Presença de lume inter ou intracelular é comum. Os mesoteliomas epiteliais apresentam bem formados desmossomos e tonofilamentos no citoplasma. Vilos muito alongados, interdigitantes contendo colágeno no pólo basal, têm sido mais associados com mesoteliomas do que adenocarcinomas, da mesma forma que a presença de glicocálices nos adenocarcinomas. Na prática, porém, essas características não são patognomônicas, podendo estar ausentes nos mesoteliomas pouco diferenciados.

Em nossa experiência, as reações histoquímicas e imuno-histoquímicas têm sido superiores no diagnóstico diferencial.

\section{OUTROS TUMORES NA EXPOSIÇÃO AO ASBESTO}

Carcinomas de laringe, esôfago, gástrico e colorretal têm sido epidemiologicamente associados com exposição ao asbesto. Porém, real associação entre asbesto e achados histológicos nunca foi demonstrada. Todos esses tumores são comuns na população em geral e apresentam várias bem-documentadas causas, como tabagismo (laringe, esôfago), álcool (laringe, esôfago), dieta (gástrico, colorretal) e classe social. Recentes estudos têm concluído que não há evidências concretas para risco elevado de qualquer desses tumores em trabalhadores expostos aos asbesto, nem há evidências para efeito doseresposta ${ }^{(43)}$. As mesmas considerações são válidas em relação às neoplasias linfoproliferativas ${ }^{(44)}$.

12. Dufresne A, Begin R, Churg A, Massé S. Mineral fibre content of lungs in mesothelioma cases seeking compensation in Québec. Am J Respir Dis Crit Care Med 1996;153:711-718.

13. Roggli VL, Greenberg SD, Pratt PC, eds. Pathology of asbestos-associated diseases. Boston: Little Brown \& Co., 1992.

14. Hillerdal G. Asbestos-related pleural disease. Semin Respir Med 1987; 9:65-74.

15. Hillerdal G, Lindgren A. Pleural plaques: correlation of autopsy findings to radiographic findings and occupational history. Eur J Respir Dis 1980;61:315-319.

16. Hillerdal G. The pathogenesis of pleural plaques and pulmonary asbestosis: possibilities and impossibilities. Eur J Respir Dis 1980;61:129138.

17. Hillerdal G, Lindgren A. Pleural plaques: correlation of autopsy findings to radiographic findings and occupational history. Eur J Respir Dis 1980;61:315-319.

18. Miller A, Tierstein AS, Selikoff IJ. Ventilatory failure due to asbestos pleurisy. Am J Med 1985;75:911-919.

19. Guidelines for the use of ILO international classification of radiographs of pneumoconioses. Rev. ed. ILO Office, 1980. (Occupational Safety and Health Series, 22).

20. Schwartz DA. New developments in asbestos induced pleural disease. Chest 1991;99:191-198.

21. Algranti E, Freitas JPB, Mendonça EMC, DeCapitani EM, Silva HC, Bussacos MA. Asbestos-related pleural thickening is independently as- 
sociated with lower levels of lung function and with shortness of breath. Inhalat Toxicol 2000;12(Suppl 3):251-260.

22. Lilis R, Miller A, Godbold J, Chan E, Selikojf IJ. Pulmonary function and pleural fibrosis: quantitative relationships with an integrative index of pleural abnormalities. Am J Ind Med 1991;20:145-161

23. Hillerdal G. Rounded atelectasis: clinical experience from 74 patients. Chest 1989; $95: 836-841$

24. Epler GR, McLoud TC, Gaensler EA. Prevalence and incidence of be nign asbestos pleural effusion in a working population. JAMA 1982 247:617-622

25. Craighead JE, Abraham JL, Churg A, Green FHY, Kleinerman J, Pratt $\mathrm{PC}$, et al. The pathology of asbestos-associated diseases of the lung and pleura cavities: diagnostic criteria and proposed grading scheme. Arch Pathol Lab Invest 1982;106:544-595.

26. American Thoracic Society. New Classification of Idiopathic Interstitial Pneumonias. Am J Respir Crit Care Med 2000.

27. Roggli VL, Pratt PC. Numbers of asbestos bodies on iron-stained sections in relation to asbestos body counts in lung tissue digests. Hum Pathol 1983;14:355-361.

28. Karjalainen A, Antilla S, Vanhala E, Vainio H. Asbestos exposure and the risk of lung cancer in a general urban population. Scand J Work Environ Health 1994;20:243-250.

29. Henderson DW, De Klerk NH, Hammar SP, et al. Asbestos and lung cancer: is it attributable to asbestosis, or to asbestos fibre burden? In: Corrin B, ed. Tumors and tumor-like disorders of the lungs. London: Churchill-Livingstone, 1997.

30. Wunsch Filho V, Moncau JE, Mirabelli D, Boffeta P. Occupational risk factors of lung cancer. Scand J Work Environ Health 1998;24:118124

31. Churg A. Asbestos, asbestosis, and lung cancer. Mod Pathol 1993;6: 509-510.

32. Roggli VL, Hammar SP, Pratt PC, Maddox JC, Legier J, Mark EJ, et al. Does asbestos or asbestosis cause carcinoma of the lung? Am J Ind Med 1994;26:835-838
33. Roggli VL. Scanning electron microscopic analysis of mineral fiber content of lung tissue in the evaluation of diffuse pulmonary fibrosis. Scanning Microsc 1991;5:71-83.

34. Browne $\mathrm{K}$. Is asbestos or asbestosis the cause of the increased risk of lung cancer in asbestos workers? Br J Ind Med 1986;43:145-149.

35. Berry G. Mortality of workers certified by pneumoconiosis medical panels as having asbestosis. Br J Ind Med 1981;38:130-137.

36. Cookson WOC, Musk AW, Glancy JJ, De Klerk NH, Yin R, Mele R, et al. Compensation, radiographic changes and survival in applicants for asbestosis compensation. Br J Ind Med 1985;42:461-468.

37. Wagner JC, Sleggs CA, Marchand P. Diffuse pleural mesothelioma and asbestos exposure in North Wertern Cape Province. Br J Ind Med 1960;17:160-171.

38. Henderson DW, Shilkin KB, Whitaker D. The pathology of mesothelioma, including immunohistology and ultrastructure. In: Henderson DW, Shilkin KB, Langlois SL, Whitaker D, eds. Malignant mesothelioma. New York: Hemisphere Publishing Corp., 1992:69-139.

39. Begin R, Gauthier JJ, Desmeules M, Ostiguy G. Work related mesothelioma in Québec 1967-1990. Am J Ind Med 1992;22:531-542.

40. Davis JM. Histogenesis and fine structure of peritoneal tumors produced in animals by injection of asbestos. J Natl Cancer Inst 1974;52: 1823-1833.

41. Daya D, McCauchey WTE. Pathology of the peritonium: a review of selected topics. Semin Diagn Pathol 1991;8:277-289.

42. Legha SS, Muggia FM. Pleural mesothelioma: clinical features and therapeutic implications. Ann Intern Med 1977;87:613-620

43. Homas DM, Garabrant DH, Gillespie BW. A meta-analysis of colorectal cancer and asbestos exposure. Am J Epidemiol 1994;139:12101222 .

44. Bengtsson N, Hardell L, Eriksson M. Asbestos exposure and malignant lymphoma. Lancet 1982;2:1463. 